\title{
Design and In-orbit Demonstration of REGULUS, an lodine electric propulsion system
}

\author{
Nicolas Bellomo ${ }^{1}$ Mirko Magarotto ${ }^{2} \mathbb{D} \cdot$ Marco Manente $^{1} \cdot$ Fabio Trezzolani $^{1} \cdot$ Riccardo Mantellato $^{1}$. \\ Lorenzo Cappellini ${ }^{1}$ - Devis Paulon ${ }^{1}$. Antonio Selmo ${ }^{1}$ - Davide Scalzi ${ }^{1} \cdot$ Marco Minute ${ }^{3} \cdot$ Matteo Duzzi $^{1}$. \\ Alessandro Barbato ${ }^{1}$ - Alessandro Schiavon ${ }^{1} \cdot$ Simone Di Fede $^{3} \cdot$ Nabil Souhair $^{4} \cdot$ Paola De Carlo ${ }^{2}$. \\ Francesco Barato $^{2} \cdot$ Fabiana Milza $^{1} \cdot$ Elena Toson $^{1} \cdot$ Daniele Pavarin $^{1,2,3}$
}

Received: 28 April 2021 / Revised: 24 May 2021 / Accepted: 25 May 2021 / Published online: 7 June 2021

(c) The Author(s) 2021

\begin{abstract}
REGULUS is an Iodine-based electric propulsion system. It has been designed and manufactured at the Italian company Technology for Propulsion and Innovation SpA (T4i). REGULUS integrates the Magnetically Enhanced Plasma Thruster (MEPT) and its subsystems, namely electronics, fluidic, and thermo-structural in a volume of $1.5 \mathrm{U}$. The mass envelope is $2.5 \mathrm{~kg}$, including propellant. REGULUS targets CubeSat platforms larger than $6 \mathrm{U}$ and CubeSat carriers. A thrust $T=0.60 \mathrm{mN}$ and a specific impulse $I_{\mathrm{sp}}=600 \mathrm{~s}$ are achieved with an input power of $P=50 \mathrm{~W}$; the nominal total impulse is $I_{\mathrm{tot}}=3000$ Ns. REGULUS has been integrated on-board of the UniSat-7 satellite and its In-orbit Demonstration (IoD) is currently ongoing. The principal topics addressed in this work are: (i) design of REGULUS, (ii) comparison of the propulsive performance obtained operating the MEPT with different propellants, namely Xenon and Iodine, (iii) qualification and acceptance tests, (iv) plume analysis, (v) the IoD.
\end{abstract}

Keywords CubeSat propulsion $\cdot$ REGULUS $\cdot$ Iodine propellant $\cdot$ Plume analysis $\cdot$ In-orbit Demonstration

\section{Introducton}

CubeSats have become increasingly common in recent years provided the dramatic reduced cost in respect to conventional satellites [1]. Combining this feature with a remarkable versatility, CubeSats allow small companies, small countries, and research centers entering the space market paving the way to a completely new paradigm. Constellations of SmallSats (namely satellites with mass $<500 \mathrm{~kg}$ ) in Low Earth Orbit (LEO) are entering the market to address the more and more demanding requirements imposed by

Mirko Magarotto

mirko.magarotto@unipd.it

1 Technology for Propulsion and Innovation SpA, Padova, Italy

2 Department of Industrial Engineering, University of Padova, Padova, Italy

3 Centre of Studies and Activities for Space "Giuseppe Colombo", University of Padova, Padova, Italy

4 Department of Industrial Engineering, University of Bologna, Forlì, Italy new applications such as global internet coverage and Internet-of-Things (IoT) [2, 3]. Nonetheless, only an on-board propulsion system enables SmallSats to fully exploit their capabilities. In fact, upcoming mission scenarios are increasingly complex since orbit change and maintenance are often required $[4,5]$. In this frame, many research centers and companies (Enpulsion, Busek, Exotrail, ThrustMe, and AVS, just to name a few) are developing new propulsion systems for CubeSats and SmallSats. The number of missions taking full advantage of a propulsion unit is still moderate, although rapidly increasing [5]. This is associated to the inherent difficulty of integrating a propulsion system into a SmallSat. In fact, a space thruster is an intrinsically complex device and strict volume, mass, power, and cost budgets overcomplicate its design.

In the STRaND-1 [6, 7] mission (launched in 2013), a water-alcohol resistojet for attitude control and a Pulsed Plasma Thruster (PPT) for orbit change were combined in a 3 U CubeSat. The BRICSat-P is a space mission in which a propulsion system has been integrated on a $1.5 \mathrm{U}$ CubeSat. It was launched in 2015, and four $\mu$ CAT thrusters [8] were used for attitude control. In the SERPENS [9] mission 
(launched in 2015) a PPT was integrated on a 3 U CubeSat for drag compensation. In 2018 a Field Emission Electric Propulsion (FEEP) has been successfully tested on orbit for the first time [10]. The In-orbit Demonstration (IoD) of the IFM Nano Thruster developed by the Austrian company Enpulsion [11] consisted in changing the semi-major axis of a LEO orbit of several meters. Finally, in 2019 the I2T5 from ThrustMe [12] was the first Iodine propelled cold gas thruster ever tested in orbit; it was integrated on a $6 \mathrm{U}$ CubeSat.

In the last decade, particular effort has been put in studying and developing Iodine-based propulsion systems targeted at CubeSats and SmallSats [12-16]. Iodine is a particularly appealing propellant because of the following properties:

- The density of Iodine is three times higher than Xenon, in fact the former can be stored at the solid state [17]; this enables a higher total impulse for the same volume of propellant.

- Iodine can be stored at moderate temperature (e.g., ambient temperature) and pressure (e.g., atmospheric pressure). Therefore no cryogenic or strict thermal control is required [13]

- Iodine has low procurement cost: $90 \%$ less than Xenon [14]

- Iodine suffers no transportation issues as the tank is not pressurized and a dedicated thermal control is not needed when the thruster is off. Therefore the entire subsystem can be shipped to the launch platform ready for use.

Moreover, Iodine and Xenon propellants guarantee comparable propulsive performances; this has been proven at least for gridded-ion and Hall-effect thrusters [14, 18]. For these reasons, the use of Iodine might be the breakthrough for the widespread diffusion of propulsion units for CubeSats and SmallSats. Nonetheless, the use of Iodine brings some challenges: (i) to avoid the condensation of the propellant along the fluidic line, the temperature of the system must be maintained at about $100{ }^{\circ} \mathrm{C}$ during operation [13]; (ii) Iodine is chemically aggressive, so materials must be selected to avoid corrosion [19]; (iii) the interactions between the Iodine plasma plume and the surfaces of the spacecraft are little known, so the risk associated to the degradation of solar arrays and optics should be carefully managed. Three planned missions that rely on an Iodine-based propulsion system are the Lunar iceCube [20], iSat [13], and Robusta$3 \mathrm{~A}$ [12]. The Lunar iceCube is a $6 \mathrm{U}$ spacecraft targeted at the observation of the Lunar surface; its launch is scheduled for late 2021. The propulsive system consists in an Iodinebased $60-\mathrm{W}$ ion thruster. The iSAT mission aims to propel a SmallSat demonstrator with a Hall-effect thruster fed with Iodine. The mission was initially planned for launch in 2017 but it is temporarily suspended because the propulsion unit needs further development. Finally, the I2T5 cold gas thruster will be used to propel the Robusta-3A CubeSat (a $3 \mathrm{U}$ technology demonstrator) whose launch is planned for late 2021.

The REGULUS propulsion unit (see Fig. 1) has been conceived by the Italian company Technology for Propulsion and Innovation (T4i) for the SmallSat market [21, 22]. The core of REGULUS is the Magnetically Enhanced Plasma Thruster (MEPT), an electric system propelled by Iodine. The propulsion unit encompasses the MEPT along with electronics, fluidic line, and thermo-structural subsystems. Its volume envelope is $1.5 \mathrm{U}$, and the total mass is $2.5 \mathrm{~kg}$ (including propellant). REGULUS targets CubeSats larger than $6 \mathrm{U}$ and CubeSat carriers. It relies on standard interfaces to ease the integration on the spacecraft, no spacegrade qualified components are used to reduce the recurring costs. REGULUS provides a thrust $T$ of $0.6 \mathrm{mN}$ and a specific impulse $I_{\text {sp }}$ of $600 \mathrm{~s}$ for an input power $P=50 \mathrm{~W}$. The nominal total impulse is $I_{\text {tot }}=3000 \mathrm{Ns}$ and it can be
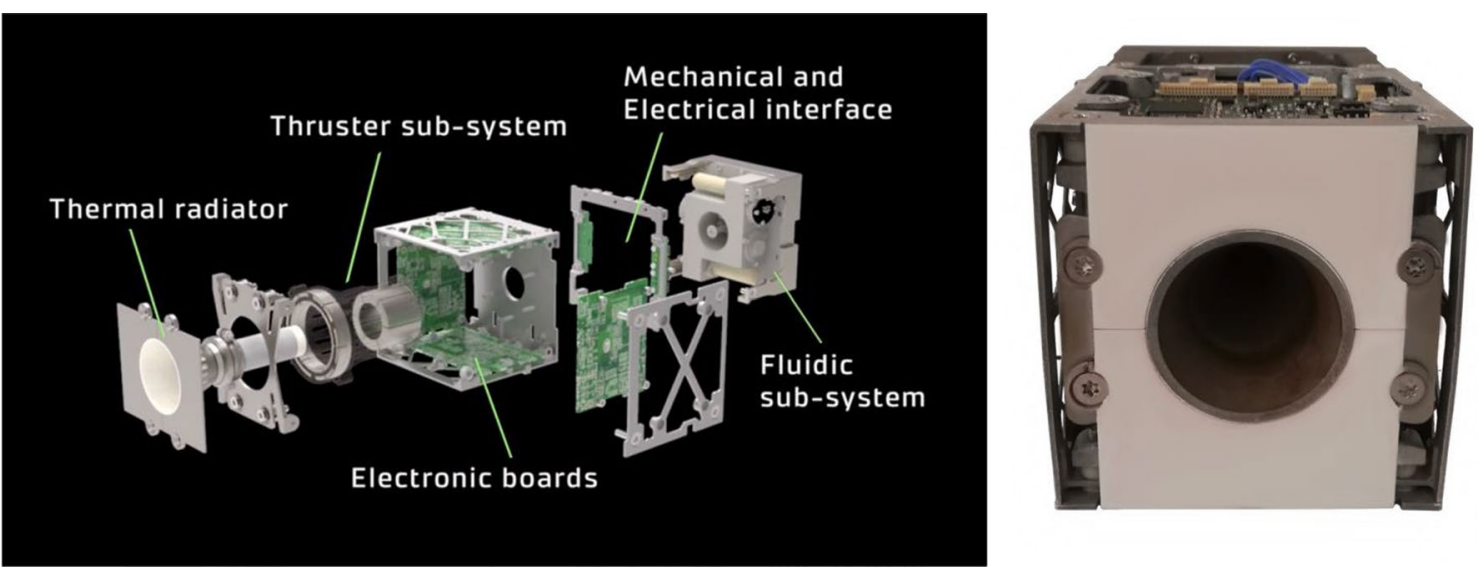

Fig. 1 Rendering (left) and picture (right) of the REGULUS propulsion unit. Subsystems have been highlighted 
increased to $I_{\text {tot }}=11,000 \mathrm{Ns}$ by enlarging the volume of the propulsive unit up to $2 \mathrm{U}$. The IoD of REGULUS is currently ongoing. The propulsive unit has been qualified, integrated on the UniSat-7 (a CubeSat carrier operated by the Italian GAUSS company) [23], and launched in March 2021 on-board of a Soyuz-2 vehicle. The objectives of the UniSat-7's mission are (i) to inject several CubeSats into a $600 \mathrm{~km}$ height Sun Synchronous Orbit (SSO), and (ii) to act as a technology demonstrator for testing specific payloads for future GAUSS missions (e.g., REGULUS). At the same time, REGULUS' primary objective is to demonstrate its capability to enhance Unisat-7 mission scenarios, while enabling maneuvers such as semi-major axis variation.

The rest of this work is organized as follows. The performance of the MEPT operated with both Xenon and Iodine propellants is discussed in Sect. 2. In Sect. 3 the subsystems of REGULUS (namely, thermo-structural, electronics and fluidics) are described and the qualification tests are illustrated. Sect. 4 is dedicated to the numerical simulation of the plasma plume to verify that the flux of charged particles impinging on the spacecraft is limited. The IoD is discussed in Sect. 5 and, finally, conclusions are drawn in Sect. 6.

\section{Magnetically enhanced plasma thruster}

The MEPT is a cathode-less plasma thruster [24, 25] specifically targeted at CubeSats (see Fig. 2). This technology is extremely appealing for space propulsion, in particular for CubeSats, because of its simple design, geometry and, in turn, reduced cost. The main characteristics of a cathode-less plasma thruster are: (i) a very simple architecture, which helps keeping at bay the cost of these systems; (ii) no electrodes in contact with the plasma neither for generation nor acceleration; (iii) possibility of operating the thruster with different propellants without a drastic redesign; (iv) absence of a neutralizer provided that the ejected plasma is currentfree and quasi-neutral. The main components of a cathodeless plasma thruster are: (i) a dielectric tube inside which the neutral gas propellant is ionized; (ii) a Radio Frequency (RF) antenna working in the $\mathrm{MHz}$ range that provides the power to produce and to heat up the plasma [26], (iii) permanent magnets that generate the magneto-static field required to enhance the plasma confinement $[27,28]$ and to improve the thrust via the magnetic nozzle effect [29-31].

The propulsive performance of the MEPT has been evaluated at the high vacuum facility of the University of Padova. To this end, a vacuum chamber of cylindrical shape (diameter $0.6 \mathrm{~m}$, length $2 \mathrm{~m}$ ) has been used [32]. A Spin HFPA-300 linear amplifier (1.8-30 MHz, power up to $300 \mathrm{~W}$ ) driven by an HP $8648 \mathrm{~B}$ signal generator provided the RF power to the MEPT. The latter was connected to the amplifier via a $50 \Omega$ coaxial cable. RF probes for vector voltage and current
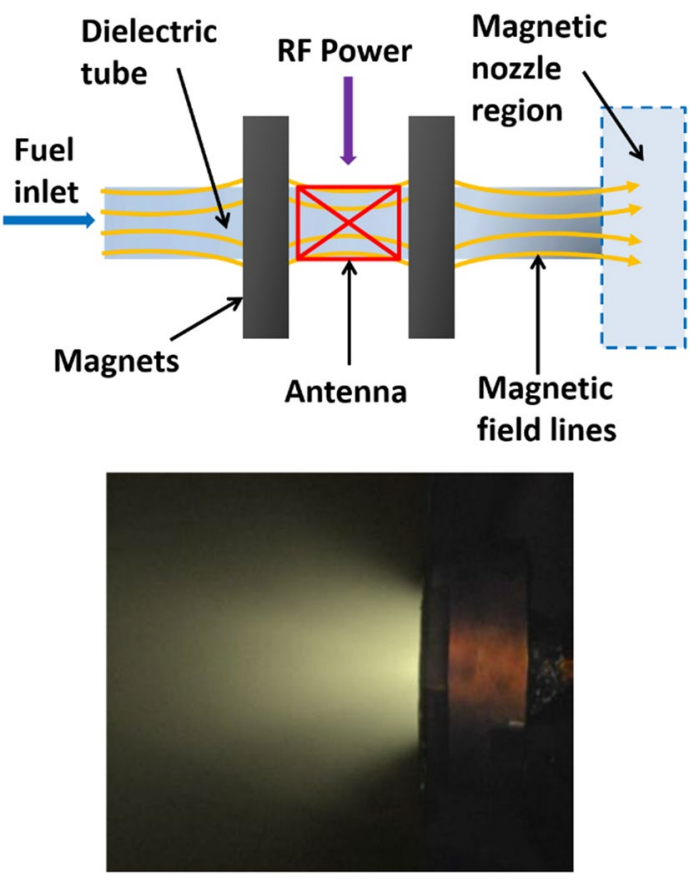

Fig. 2 Schematic (above) and picture (below) of the MEPT integrated into REGULUS. In the picture, the thruster is being tested with Iodine propellant: input power $P=50 \mathrm{~W}$, mass flow rate $0.10 \mathrm{mg} / \mathrm{s}$

measurement have been used to characterize the electrical power coupled to the antenna [21]. The MEPT has been propelled both with Xenon and Iodine. The mass flow rate of Xenon has been controlled via a MKS 1179B regulator connected to a pressurized reservoir. Instead, the Iodine feeding line consisted of a heated tank and a manifold comprising valves and mass flow control orifices [33]. The thrust generated by the MEPT has been measured with a counterbalanced pendulum specifically designed for RF thrusters of small-to-medium size [34]. The confidence interval for each measurement is: (i) $\pm 15-20 \%$ for thrust, (ii) $\pm 10 \%$ for the power provided to the MEPT, along with (iii) few percent points for the Xenon mass flow rate, and $\pm 10-15 \%$ for Iodine. These values depend on the accuracy of each instrument and on measurement methods. Specifically, the uncertainty on the thrust is mainly associated to calibration errors (this procedure is performed with known masses), and the correction of the thermal drifts induced on the balance (see [34] for more details). Errors on phasing voltage and current probes result in $\pm 10 \%$ uncertainty for the electrical power. The uncertainty on Iodine mass flow rate is due to the pressure-based control strategy adopted for this test (see [33] for more details). Finally, the error bars associated to indirect measures, as for the thruster efficiency and the thrust-to-power ratio (see Fig. 3), are computed with the uncertainty propagation law [34].

In Fig. 3, the thrust efficiency $\eta$ and the power-to-thrust ratio $T / P$ are depicted as functions of the input power $P$ 

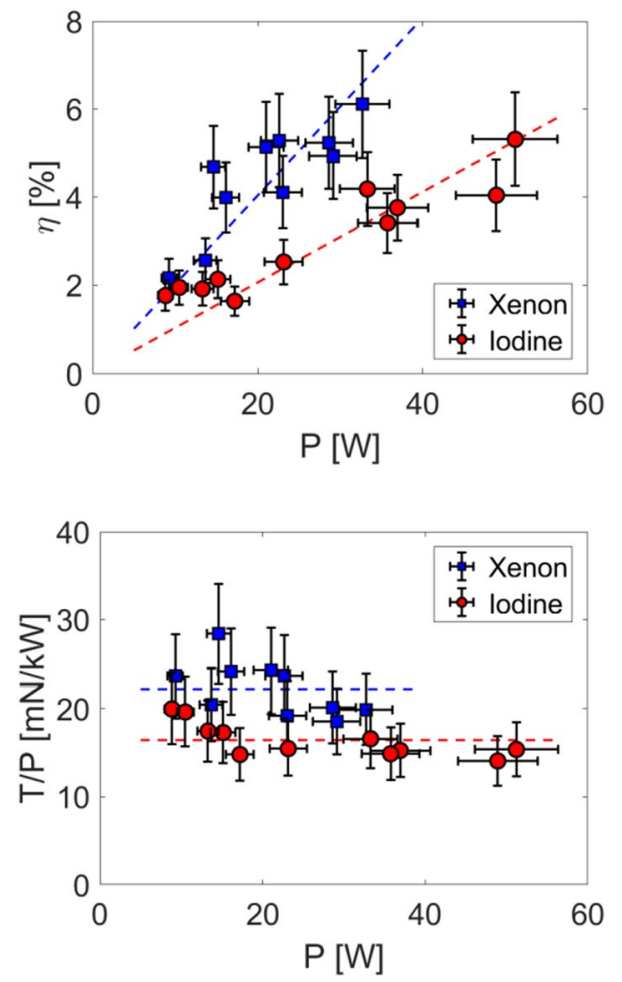

Fig. 3 Comparison of the propulsive performance when the MEPT is operated with Xenon and Iodine propellants. Thruster efficiency $\eta$ (above), and thrust-to-power ratio $T / P$ (below) as functions of the input power to the thruster $P$. The mass flow rate is $0.10 \mathrm{mg} / \mathrm{s}$

namely the power absorbed by the thruster (thanks to the good impedance matching, the power reflected is lower than $5 \%)$. In particular:

$\eta=\frac{g_{0} T I_{\mathrm{sp}}}{2 P}$,

where $T$ is the thrust, $I_{\mathrm{sp}}$ the specific impulse, and $g_{0}$ the standard gravity. The working frequency of the RF antenna was fixed at $2 \mathrm{MHz}$ and $P$ has been varied in the range $15-60 \mathrm{~W}$. The vacuum chamber pressure was maintained at $10^{-3} \mathrm{~Pa}$, and the MEPT was operated with $0.10 \mathrm{mg} / \mathrm{s}$ mass flow rate of both Xenon and Iodine propellants. The efficiency $\eta$ is a linear functions of $P$, while the maximum value achieved with Iodine is about $5 \%$ and $6 \%$ with Xenon. Those values are in line with other cathode-less thrusters not subject to strict power and volume budgets [35]. The thrust-to-power ratio $T / P$ is almost independent from $P$. Iodine presents a poorer performance compared to Xenon, being T/P 20\% lower operating the MEPT with the former propellant than with the latter. This difference seems to be due to the molecular form of Iodine $\left(I_{2}\right)$, since part of the available power is lost into dissociation along with excitation of vibration and rotation modes instead of ionization.
Table 1 Maximum performance evaluated during the test campaign

\begin{tabular}{llll}
\hline Propellant & $T[\mathrm{mN}]$ & $I_{\mathrm{sp}}[\mathrm{s}]$ & $P[\mathrm{~W}]$ \\
\hline Xenon & 0.63 & 630 & 32 \\
Iodine & 0.77 & 770 & 51 \\
\hline
\end{tabular}

Thrust $T$, specific impulse $I_{\mathrm{sp}}$ and power absorbed by the thruster $P$. The mass flow rate is $0.10 \mathrm{mg} / \mathrm{s}$

The maximum $T$ and $I_{\mathrm{sp}}$ measured with each propellant are reported in Table 1. Further results regarding the testing campaign can be found in [36] (in particular for what discussions on $T$ and $I_{\mathrm{sp}}$ are concerned).

In conclusion, Iodine is demonstrated as a valid candidate to propel CubeSat missions provided the advantages offered at system level (e.g., capability of being stored at solid state) and the propulsive performance only $20 \%$ lower, in terms of $T / P$, in respect to Xenon.

\section{Regulus subsystems}

\subsection{Thermo-structural}

The thermo-structural subsystem has three main functions: (i) to provide a sufficiently stiff structural frame for the MEPT, the fluidic line, and the electronics, (ii) to dissipate the heat produced within the REGULUS unit so that each component is maintained within the correct temperature range, and (iii) to provide a thermo-mechanical interface with the satellite. A more detailed list of requirements is reported in Table 2.

One of the key aspects to satisfy the strict mass and volume budgets ( $2.5 \mathrm{~kg}$ and $1.5 \mathrm{U}$, respectively) is the extensive adoption of the additive manufacturing technique. Specifically, this production process allows: (i) to manufacture shapes that would be impossible with traditional approaches and (ii) to reduce bolted junctions increasing the reliability of the system. In addition, a mix of materials has been used to develop a sufficiently stiff system capable of withstanding the harsh vibrational environment during launch operations. Materials as Scalmalloy and Ti-6Al-4V [40] guarantee high strength, stiffness and lightness. The structural design of REGULUS has been driven by semi-analytical calculations and Finite Element Method (FEM) analyses, performed with the Ansys software [41]. At the same time, sinusoidal and random vibration tests have been performed to verify the design and to qualify the propulsion unit. The measurement campaign has been performed at the facilities of the University of Padova (see Fig. 4) assuming the spectra of the Soyuz launcher. The first natural frequency of the REGULUS unit is about $250 \mathrm{~Hz}$ and a good margin of safety against damages has been found. Moreover, the accordance between the 
Table 2 Thermal, mechanical and interface requirements considered in the design of REGULUS
Mechanical requirements

1. The mass of REGULUS shall be lower than $2.5 \mathrm{~kg}$ and its volume envelope within $1.5 \mathrm{U}$

2. The first natural frequency of the system shall be higher than $200 \mathrm{~Hz}$

3. REGULUS shall be compliant with the vibrational environment of the main launch systems such as Ariane 5, Vega, Falcon 9, and Soyuz [37]

Thermal requirements

1. REGULUS shall withstand an operative temperature environment ranging from -25 up to $+70{ }^{\circ} \mathrm{C}$

2. The maximum heat to be dissipated is assumed equal to $60 \mathrm{~W}$

3 . The heat shall be dissipated relying only on a radiator mounted on the nozzle surface of REGULUS

4. The temperature of the electronics subsystem shall not exceed $+85^{\circ} \mathrm{C}$

5. The temperature of the fluidic line shall be maintained at $+95^{\circ} \mathrm{C}$ during operations

Interface requirements

1. REGULUS shall fit the main CubeSat structure such as Endurosat, Gomspace and ISIS [38, 39]

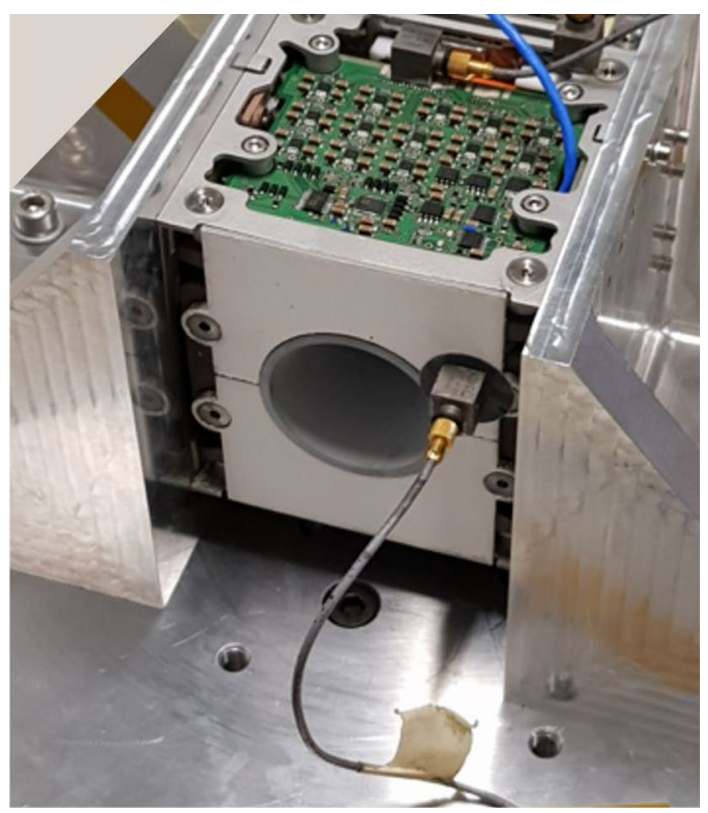

Fig. 4 Mechanical vibration test of the Electro-Magnetic model of REGULUS

results of the FEM simulations and the real behavior of the propulsion unit is good (e.g., numerical and experimental estimations of the first natural frequency differ by $10 \mathrm{~Hz}$ ). After the vibration tests, each component of the REGULUS unit has been inspected and subsequent functional tests have been performed: each electronic board, valve, heater, temperature and pressure sensor has been checked. Finally, the nominal operation of the entire propulsion unit has been verified. Since no structural or functional failure has been detected, the mechanical design was considered successfully qualified.

To satisfy the thermal requirements of each component, two thermal paths have been designed, namely the high temperature and the low temperature one (see Fig. 5). Dedicated low emittance coatings have been used to insulate the two paths. The MEPT and the radiator constitute the

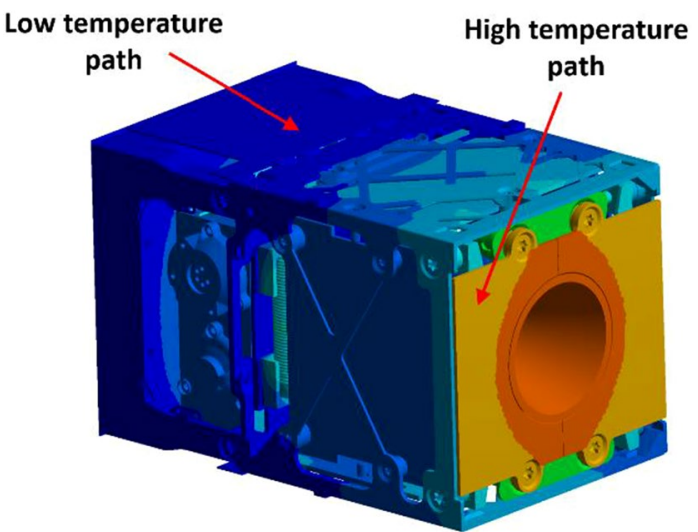

Fig. 5 Schematic of the thermal control system; thermal paths highlighted

high temperature path; the former is the principal source of thermal power and the latter is used for the dissipation of the heat into space. The electronics and the fluidic line are included in the low thermal path that is interfaced with the satellite to dissipate the heat produced by the subsystems of REGULUS. The thermal design has been driven by semi-analytical models and FEM analyses. In particular, two scenarios have been considered: (i) the Worst Hot Case (WHC), where the satellite is subject to the solar flux and its temperature is $+70{ }^{\circ} \mathrm{C}$ and (ii) the Worst Cold Case (WCC), namely no solar flux and the satellite at $-25^{\circ} \mathrm{C}$. The assumed amount of power to be dissipated is $60 \mathrm{~W}$ because of the heat produced during operations and about $3 \mathrm{~W}$ due to the solar flux. The results of the thermal analysis for the WHC are shown in Table 3 in terms of temperature of the main critical components. Specifically, data retrieved with a FEM analysis and a Lumped Parameter Model (LPM) created with the Octave software [42] are reported. The latter is intended to cross-validate the FEM outputs and to obtain results in a shorter time. The agreement between the two models is satisfactory being the differences registered in the order of $5-10^{\circ} \mathrm{C}$. Finally, thermo-vacuum tests 
Table 3 WHC maximum temperature of critical components; comparison between Finite Elements Method (FEM) and Lumped Parameters Model (LPM)

\begin{tabular}{lll}
\hline Component & $\begin{array}{l}\text { Temperature }\left[{ }^{\circ} \mathrm{C}\right]- \\
\text { FEM model }\end{array}$ & $\begin{array}{l}\text { Temperature } \\
{\left[{ }^{\circ} \mathrm{C}\right]-\mathrm{LPM}} \\
\text { model }\end{array}$ \\
\hline Discharge chamber & 355 & 351 \\
Radiator & 273 & 279 \\
Electronics & 87 & 90 \\
Fluidics & 84 & 92 \\
Frame & 85 & 87 \\
Interface & 73 & 75 \\
\hline
\end{tabular}

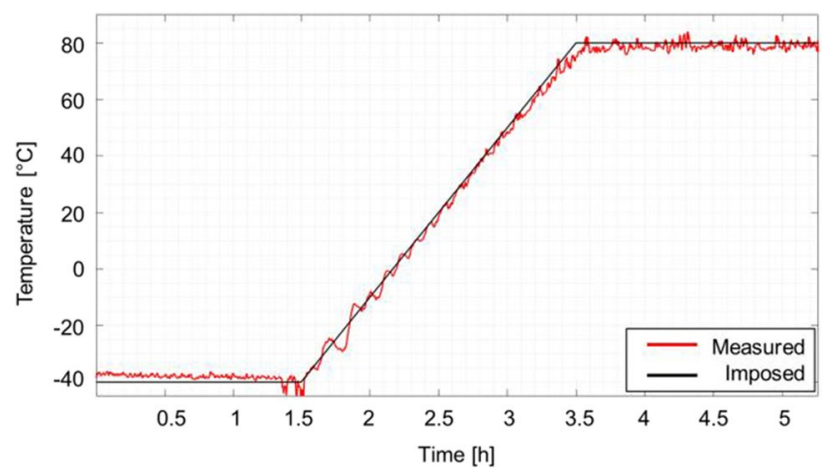

Fig. 6 Temperature profile of thermo-vacuum tests

have been performed at the facilities of the University of Padova to qualify the propulsion unit and to validate the numerical models. REGULUS has been tested inside a dedicated Thermo VAcuum Chamber (TVAC) at a pressure $8 \times 10^{-1} \mathrm{~Pa}$. The temperature has been varied from -40 to $80{ }^{\circ} \mathrm{C}$ for four times (see Fig. 6). After the thermo-vacuum cycles, the subsystems have been inspected and functional tests have been performed. No failure has been detected, and so the thermal design was considered successfully qualified.

In conclusion, an interface module has been created that includes all mechanical, thermal and electrical connections. The module is compatible with standard CubeSat structures $[38,39]$.

\subsection{Electronics}

The electronics have been designed to meet the budget requirements typical of the CubeSat application while maintaining competitive standards of reliability. To this end, Components Off-The-Shelf (COST) have been largely used. This was not detrimental in terms of reliability provided that, according to the European Cooperation for Space Standardization (ECSS), the selection of COTS was driven by a dedicated risk analysis. First, an average mission duration of 3-5 years and a LEO of altitude about $600 \mathrm{~km}$ have been assumed to evaluate the expected radiation environment. Second, calculations with the SPENVIS tool [43] provided a requirement in terms of Total Ionization Dose (TID) that was not highly demanding for the mission at hand. Third, COTS compliant with the expected radiation environment have been selected to design the electronics subsystem. In particular, the approach of careful COTS design proposed by Sinclair [44], the ECSS_E_ST-10-12C, and the ECSSQ-ST-60C have been referenced for this analysis. It is worth specifying that electronics are fully compliant with the mission scenario of the IoD. Moreover, the selection of COTS can be tailored to different customers' needs by means of a dedicated risk analysis. The electronics rely on a flexible architecture and they have been designed for operating REGULUS with an input power from 30 up to $60 \mathrm{~W}$, although the nominal value is $50 \mathrm{~W}$. Moreover, a simplified laboratory version of the electronics subsystem has been used in a completely different application field, namely plasma antennas [45-48]. The schematic of the electronics of REGULUS is depicted in Fig. 7.

\subsubsection{Interface}

The electronics have been interfaced with the satellite by means of four types of connectors that differ from one another in terms of size and number of pins (to avoid mistakes):

- an "insert before flight" type connector is included for safety reasons;

- two separated communication lines (referred to as primary and secondary) that rely on two redundant CANbus and one I2C [49] protocols are used for redundancy;

- one power connector is used for feeding the electronics with the $12 \mathrm{~V} \mathrm{DC}$ power from the bus.

The connectors are interfaced to the structure by means of two Printed Circuit Boards (PCBs). Finally, a JTAG connector [50] is easily reachable also after integration for updating the software.

\subsubsection{Software}

The control software is based on FreeRTOS [51] and manages the following specific tasks:

- controlling the thruster in terms of thermal regulation, mass flow, and electrical power;

- managing the housekeeping and the thrust operational states;

- communicating with the satellite through CANbus or $\mathrm{I} 2 \mathrm{C}$. 
Fig. 7 Schematic of the electronics subsystem of REGULUS

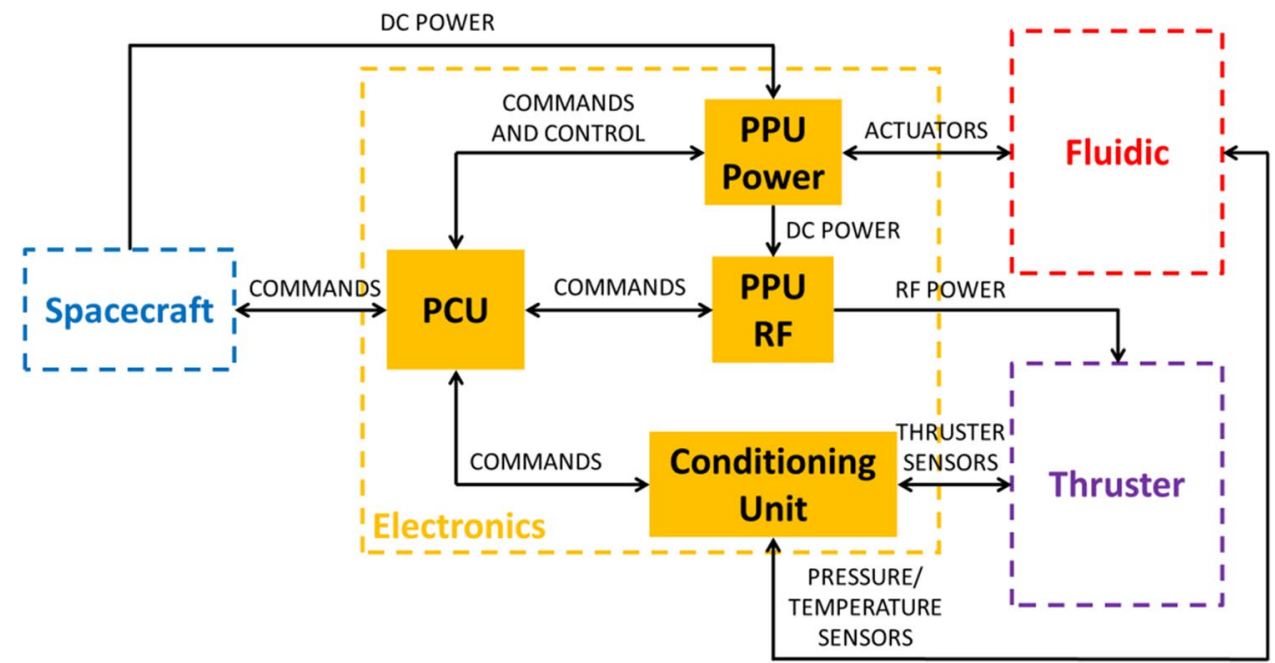

\subsubsection{Architecture}

The electronics subsystem consists of four boards disposed around the MEPT, thermally and electrically insulated from it.

The Power Control Unit (PCU) controls and monitors the operations of REGULUS and provides the interface with the spacecraft via CANbus and/or I2C. More in detail, the PCU:

- is the interface with the spacecraft for the data link receiving telecommands and sending telemetries;

- processes the data for the thermal control;

- runs algorithms for thruster ignition and shut down;

- monitors the status of the thruster and manages the Failure Detection, Isolation, and Recovery (FDIR).

The PCU is reprogrammable in flight thanks to the bootstrap functionality and an external flash memory in which the software sent via data link can be stored.

The Power Processing Unit (PPU)—Power provides DC power to sensors, valves and heaters involved in the control of the fluidic line, along with it feeds the DC power to the PPU-RF.

The $P P U-R F$ is the DC/AC converter that provides the $\mathrm{RF}$ power to supply the MEPT. It is the main power segment of the electronics subsystem.

The Conditioning Unit provides the signal conditioning for the diagnostics of REGULUS.

\subsubsection{Testing}

Successful functional tests of the electronics have been performed in vacuum both at ambient temperature $\left(20 / 30{ }^{\circ} \mathrm{C}\right)$ and in a variable temperature environment $\left(-20 / 50{ }^{\circ} \mathrm{C}\right)$. Moreover, Electro-Magnetic Compatibility (EMC) tests, compliant with ECSS, have been performed integrating
REGULUS on the CubeSat Test Platform (CTP) of the Polytechnic University of Torino [52]. The RF emissions and the generated electro-magnetic fields have produced noise values up to the $15 \mathrm{dBm}$ without affecting the functionalities of both the CTP and REGULUS. Moreover, the noise generated by the propulsion system on the power line is negligible $(<1 \mathrm{dBm})$.

\subsection{Fluidic line}

The main requirement of the fluidic subsystem of REGULUS (see Fig. 8) is to deliver a $0.10 \mathrm{mg} / \mathrm{s}$ mass flow rate of Iodine during thruster operation. To obtain a stable propulsive performance, the tolerance on the delivered mass flow rate is $\pm 5 \%$ throughout the overall mission duration. The fluidic subsystem is composed of a tank where the propellant is stored in solid state, a manifold that acts as flow regulator, and an injector that interfaces the subsystem with the MEPT. The additive manufacturing technique has been extensively adopted to achieve compliance with strict volume and mass budgets. A careful selection of the material for the components wetted by Iodine has been accomplished in order to avoid chemical corrosion. The hardware is made from Nickel superalloys such as Inconel and Hastelloy, whereas

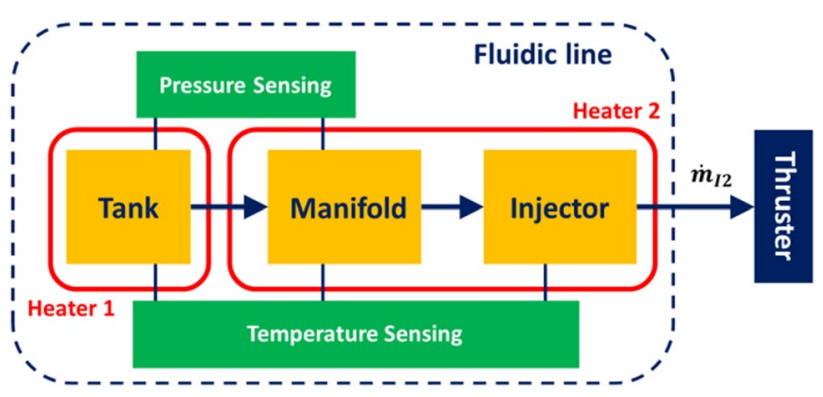

Fig. 8 Schematic of the fluidic line of REGULUS 
fluoroelastomer (i.e., FKM and FFKM) and polytetrafluoroethylene (PTFE) [53] sealing is adopted at interfaces. The dimension of the tank, can be varied, according to the mission needs, independently from the rest of the propulsive unit. When the tank is heated, the solid Iodine sublimates and enters the manifold. The latter is the technological core of the fluidic subsystem and maintains the nominal mass flow rate. It comprises a pair of valves along with pressure and temperature transducers controlled in closed-loop. The injector has two main functions: (i) it tunes the thermal resistance between the hot thruster and the fluidic line and (ii) it delivers the gaseous Iodine to the MEPT where it is turned into plasma. Two thin-foil heaters are included in the fluidic line to control the temperature of the subsystem via a closed-loop strategy. Specifically, the thermal control allows to: (i) ensure the sublimation of the solid Iodine into the tank, (ii) perform a coarse control of the mass flow rate, (iii) avoid the obstruction of the fluidic line due to the recondensation of the Iodine, (iv) avoid the liquid state of the Iodine (temperature $<115^{\circ} \mathrm{C}$ everywhere), and (v) avoid the over-heating of the entire system with the consequent failure of the electronics.

Finally, the fluidic line has been tested in order to verify its capability to provide a $0.10 \mathrm{mg} / \mathrm{s}$ Iodine mass flow rate with accuracy of $\pm 5 \%$ [33]. Vacuum tests have been performed showing that the line was compliant with nominal specifications.

\section{Plume analysis}

The effects of the iodine plasma plume on the surfaces of the spacecraft are not well-known, thus it is worth assessing that these interactions are minimized. In the following, a preliminary analysis performed with the numerical solver Spacecraft Plasma Interaction System (SPIS) [54] is presented.

The plume generated by the MEPT has been simulated with a 3-Dimensional (3D) Particle-In-Cell (PIC) approach in order to carefully account for the non-Maxwellian dynamics of the electrons [55]. The computational domain consists of a cylinder $16 \mathrm{~cm}$ in diameter and $16 \mathrm{~cm}$ in length (see Fig. 9). According to a previous work, the domain is sufficiently large to grasp the principal phenomena governing the plasma expansion (e.g., demagnetization of both ions and electrons) [56]. The spacecraft (a 6 U CubeSat) is an equipotential surface only partially included within the computational domain. This assumption aims at reducing the intense computational cost of PIC simulations and its rationale has been discussed more in detail in the following. The species simulated are electrons $\left(\mathrm{e}^{-}\right)$and ions $\left(\mathrm{I}^{+}\right)$produced by the thruster. Both neutral particles (e.g., $I_{2}$ and $I$ ) and other ionic species (e.g., $I_{2}^{+}$and $I^{-}$) have been neglected provided that they are expected to be present in minor percentage [57]. The
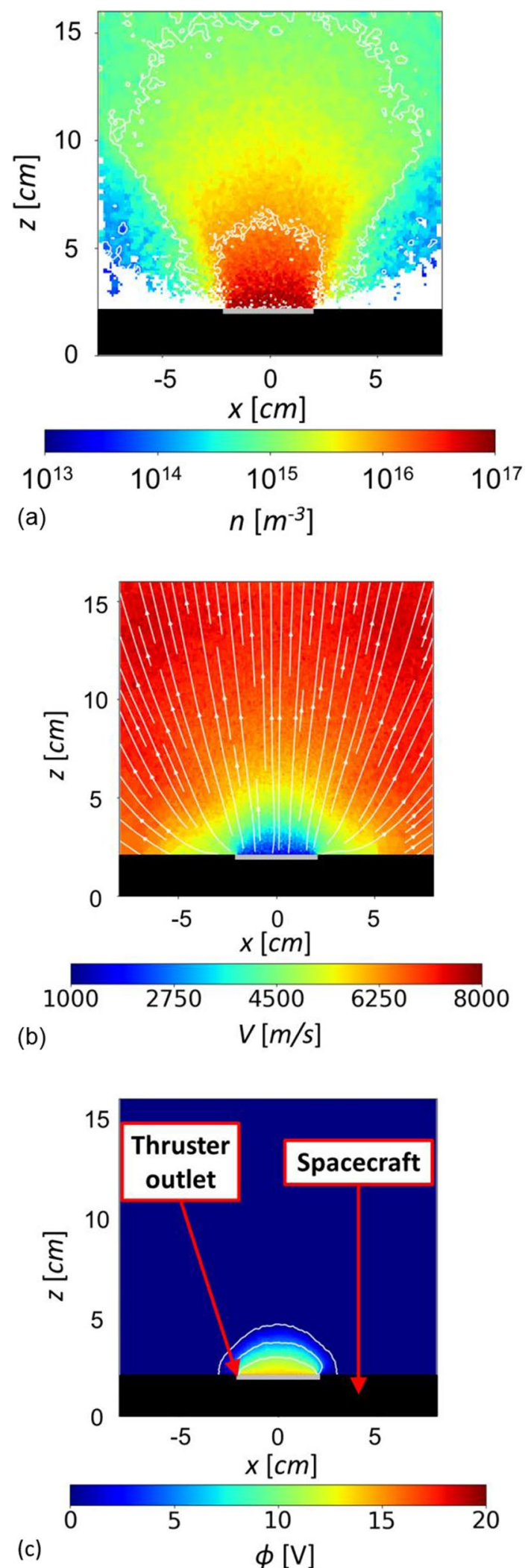

Fig. 9 PIC simulation of the plume: a plasma density $\mathbf{n}, \mathbf{b}$ ions speed $\mathrm{V}$, and $\mathbf{c}$ plasma potential $\phi$ as functions of the position ( $x$ and $z$ coordinates). Highlighted the location of the spacecraft and of the thruster outlet 
plume is assumed non-collisional and the ambient plasma has been neglected. The latter assumption seems reasonable provided that the plume produced by REGULUS has density and temperature orders of magnitude higher in respect to the ambient plasma [58]. Particles are injected in the computational domain from the surface labeled "Thruster outlet" in Fig. 9. The following properties are assumed [30]

- Ion temperature $T_{i}=0.3 \mathrm{eV}$, electron temperature $T_{\mathrm{e}}=3 \mathrm{eV}$

- Ions and electrons injection flux $\Gamma=5 \times 10^{20} \mathrm{~m}^{-2} \mathrm{~s}^{-1}$

- Ions speed $V_{0}=1370 \mathrm{~m} / \mathrm{s}$

The value of $\Gamma$ derives from the assumption of $100 \%$ propellant utilization $\left(0.10 \mathrm{mg} / \mathrm{s}\right.$ mass flow rate), and $V_{0}$ is the Bohm speed. Further details on boundary conditions and the methodology to compute self-consistently the equilibrium potentials are discussed in [56]. Finally, to reduce the simulation time, the vacuum permittivity has been reduced of a factor $f=400$ in respect to the real value in accordance with the similarity laws reported in [59].

In Fig. 9 maps of plasma density $n$, ion speed $V$, and potential $\phi$ are depicted as a function of the position $(x-z$ coordinates). At the location of the thruster outlet, the plasma density peaks as $n \approx 10^{17} \mathrm{~m}^{-3}$. This value decreases of roughly two orders of magnitudes at the external boundary. This decrement is far more intense for what the surface of the spacecraft is concerned (more than four orders of magnitude). The magnitude of $V$ increases in the plume downstream of the thruster outlet, as expected in a magnetic nozzle [60]. The maximum value is $V \approx 8000 \mathrm{~m} / \mathrm{s}$ (almost 5 times higher than $V_{0}$ ) at the external boundary. Interestingly, the trajectories of the ions seem to be very mildly affected by the magneto-static field (negligible gyration motion), thus they can be considered demagnetized in the overall domain. The plume is acceptably collimated: $95 \%$ of the exhausted particles flux is enclosed in an aperture with a half-angle of $30^{\circ}$. Less than $0.1 \%$ of the particles impinges on the satellite. Specifically, $90 \%$ of the ions colliding against the spacecraft are collected on the surface of the heater. The plasma potential at the thruster outlet is $\phi \approx 20 \mathrm{~V}$. Considering that at an infinite distance from the thruster outlet $\phi$ is assumed to be at $0 \mathrm{~V}$, the potential drop across the plume is comparable to the one expected across a plasma sheath [60]. The equilibrium potential of the satellite is $-0.5 \mathrm{~V}$. In Fig. 10, the trajectories of a selection of electrons that collide against the spacecraft is depicted. In contrast to the ions, electrons describe a more disordered motion due to their higher temperature. It is, however, possible to distinguish clearly the Larmor rotations described by the particles that are frozen to the magneto-static field lines. Notably, few centimeters downstream the thruster outlet, electrons detach from the field lines and their motion becomes completely chaotic.

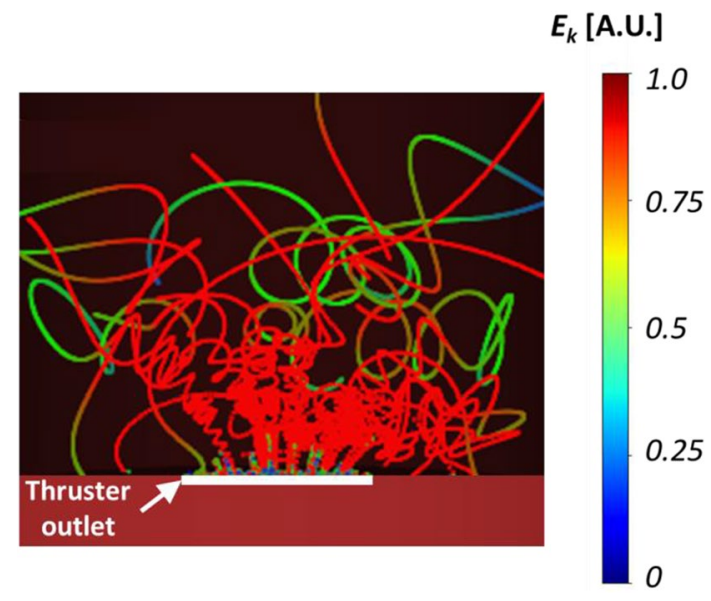

Fig. 10 Trajectories of electrons colliding with the spacecraft. Color of the trajectory depends on the local kinetic energy of the particle $E_{\mathrm{k}}$

This happens roughly when the plasma potential is $2-3 \mathrm{~V}$. This result enforces the assumption of considering only a portion of the spacecraft in the simulation domain, in fact a very reduced number of charged particles are expected to stay frozen to field lines resulting in intense and localized interactions with the surfaces of the spacecraft placed out of the computational domain. Finally, it is interesting to notice that the kinetic energy of the electrons $\left(E_{\mathrm{k}}\right)$ rapidly decreases downstream the thruster outlet, in agreement with theoretical models of a magnetic nozzle [35, 60].

The main result of this preliminary analysis is that the interactions between the plasma plume of REGULUS and the satellite are comparable with other electric thrusters (e.g., Hall-effect systems [61]). As a result a major degradation of the surfaces of the spacecraft is not expected provided that the region near the thruster outlet (i.e., the radiator) is realized with materials compatible with iodine. Nonetheless, more detailed investigations are required to account for plasma collisions within the plume, the ambient plasma, and the different materials that constitute a realistic spacecraft. Moreover, an enlarged computational domain including the overall spacecraft will be considered to cross validate the present results.

\section{In-orbit Demonstration}

REGULUS has been integrated on-board of the Unisat-7 satellite developed by the Italian company GAUSS [23]. Before integration, sinusoidal and random vibrations along with thermo-vacuum (temperature range $-20 / 50{ }^{\circ} \mathrm{C}$ ) tests have been performed for the acceptance. The launch has been successfully accomplished in March 2021 on-board of a Soyuz-2 vehicle. UniSat-7 is a CubeSat carrier and a technology demonstrator for payloads as the avionic system 
Table 4 Orbital parameters of UniSat-7

\begin{tabular}{ll}
\hline Launch vehicle & Soyuz-2 \\
Launch base & Baikonur \\
Launch date & March 22nd 2021 \\
Orbit & Sun Synchronous \\
Altitude & $\approx 600 \mathrm{~km}$ \\
Eccentricity & $\approx 0.005$ \\
\hline
\end{tabular}

DeCAS [62] and REGULUS. The target orbit is circular, Sun-Synchronous, and has height $600 \mathrm{~km}$ (see Table 4 for further details). One of the main objectives of the current mission is to test the capability of REGULUS to improve the mobility of UniSat-7. The injection of CubeSats on different orbits (e.g., at various altitudes) will be simulated and the decommissioning of UniSat-7 will be verified as final task of REGULUS. Currently, internal checks to understand the interactions of the subsystems with the space environment and the satellite are ongoing. For example, the capability to heat up the propellant and the tank in different conditions (e.g. starting from different initial temperatures) is under analysis. After this, a series of maneuvers will be performed with incremental thrust duration, from minutes up to hours (potentially, REGULUS can be operated also during eclipse time thanks to the battery pack of UniSat-7). Finally, a longer thrust will allow to verify the performance of the propulsive unit and, in turn, the capability of REGULUS to decommission UniSat-7. The former will be estimated from telemetry data, specifically the thrust can be derived from the altitude change (expected of several kilometers) produced by the continuous firing of REGULUS.

\section{Conclusions}

This work is devoted to the description of the design, the performance, and the IoD of REGULUS. The latter is an Iodine-propelled electric propulsion unit conceived at the Italian company T4i. REGULUS targets CubeSats larger than $6 \mathrm{U}$ and CubeSat carries. It has a mass of $2.5 \mathrm{~kg}$ and an envelope of $1.5 \mathrm{U}$. The MEPT (a RF cathode-less thruster) is the core of REGULUS which also includes the thermostructural, the electronic and the fluidic subsystems. The propulsive performance has been evaluated while feeding the MEPT with Xenon and Iodine propellants. Comparable values of thruster efficiency $\eta \approx 5 \%$ and thrust-to-power ratio $T / P \approx 20 \mathrm{mN} / \mathrm{kW}$ have been measured for an input power $P=50 \mathrm{~W}$. These values are in line with other cathode-less thrusters not subject to strict volume and power budgets [35]. Successful sinusoidal and random vibration along with thermo-vacuum (temperature range $-40 / 80{ }^{\circ} \mathrm{C}$ ) qualification and acceptance tests have been performed before integrating REGULUS on-board of the UniSat-7 satellite for its IoD. Moreover, functional tests have been performed on the complete system both at ambient temperature $\left(20 / 30^{\circ} \mathrm{C}\right)$ and in a variable temperature environment $\left(-20 / 50^{\circ} \mathrm{C}\right)$. The interactions between the iodine plasma plume and the surfaces of the spacecraft have been preliminarily evaluated by means of the SPIS solver. The latter seem in line with other electric thrusters (e.g., Hall-effect systems [61]). Finally, REGULUS has been launched in March 2021 on board of a Soyuz-2 vehicle for its IoD that is currently on-going.

Funding Open access funding provided by Università degli Studi di Padova within the CRUI-CARE Agreement.

Open Access This article is licensed under a Creative Commons Attribution 4.0 International License, which permits use, sharing, adaptation, distribution and reproduction in any medium or format, as long as you give appropriate credit to the original author(s) and the source, provide a link to the Creative Commons licence, and indicate if changes were made. The images or other third party material in this article are included in the article's Creative Commons licence, unless indicated otherwise in a credit line to the material. If material is not included in the article's Creative Commons licence and your intended use is not permitted by statutory regulation or exceeds the permitted use, you will need to obtain permission directly from the copyright holder. To view a copy of this licence, visit http://creativecommons.org/licenses/by/4.0/.

\section{References}

1. Villela, T., et al.: Towards the thousandth CubeSat: a statistical overview. Int. J. Aerosp. Eng. (2019). https://doi.org/10.1155/ 2019/5063145

2. Straub, J., Gill, E., Sundaramoorthy, P., Bouwmeester, J., Zandbergen, B., Reinhard, R.: Formation flying within a constellation of nano-satellites: The QB50 mission. Acta Astronaut. 82(1), 110-117 (2013)

3. Buchen, E.: Small satellite market observations. SSC15-VII-7, 29th AIAA/USU small satellite conference, Logan, UT, USA (2015)

4. Mueller, J., Hofer, R., Ziemer J.: Survey of propulsion technologies applicable to cubesats. Proceedings of the Joint Army-navyNASA-air Force (JANNAF), Colorado Springs, CO, USA (2010)

5. Poghosyan, A., Golkar, A.: Cubesat evolution: analyzing CubeSat capabilities for conducting science missions. Prog. Aerosp. Sci. 88, 59-83 (2017)

6. Bridges, C.P., Kenyon, S., Shaw, P., Simons, E., Visagie, L., Theodorou, T., Yeomans, B., Parsons, J., Lappas, V., Underwood, C. et al.: A baptism of fire: The STRaND-1 nanosatellite. SSC13X-3, 28th AIAA/USU Small Satellites Conference, Logan, UT, USA (2013)

7. Bridges, C., Kenyon, S., Underwood, C., Lappas, V.: STRaND-1: The world's first smartphone nanosatellite. 2nd IEEE international conference on space technology (ICST), Athens, Greece (2011)

8. Hurley, S., Teel, G., Lukas, J., Haque, S., Keidar, M., Dinelli, C., Kang, J.: Thruster subsystem for the United States Naval Academy's (USNA) ballistically reinforced communication satellite (BRICSat-P). Trans. Jpn. Soc. Aeronaut. Space Sci. Aerosp. Technol. Jpn. 14(30), 157-163 (2016)

9. de Brum, A.G.V., Baroni, L., da Silva, A.L., Coelho, F.S.N., Ferreira, E.S., Zanardi, M.C., Spengler, A.W.: Attitude control system 
proposed for SERPENS-2 space mission. Comput. Appl. Math. 37, 4743-4756 (2018)

10. Krejci, D., Reissner, A., Seifert, B., Jelem, D., Horbe, T., Plesescu, F., Friedhoff, P., Lai, S.: Demonstration of the IFM nano feep thruster in low eath orbit. 4S symposium, Sorrento, Italy, 2018

11. Official Enpulsion website: https://www.enpulsion.com/ (2020). Accessed 9 Sept 2020

12. Martinez, J.M. et al.: An off-axis iodine propulsion system for the robusta-3A mission. SSC20-IX-03, 34th AIAA/USU conference on small satellites, Logan, UT, USA, 2020

13. Polzin, K.A., Seixal, J.F., Mauro, S.L., Burt, A.O., Martinez, A., Martin, A.K.: The iodine satellite (iSat) propellant feed systemdesign and development. IEPC-2017-11, 35th international electric propulsion conference, Atlanta, GA, USA (2017)

14. Szabo, J., Robin, M., Paintal, S., Pote, B., Hruby, V., Freeman, C.: Iodine propellant space propulsion. IEPC-2013-311, 33th international electric propulsion conference, Washington, DC, USA (2013)

15. Paganucci, F. Saravia, M.M., Mininni, M., Bernazzani, L., Ceccarini, A., Boulzaguet, T., Pellegrini, G., Ducci, C., Pedrini, D., Andreussi, T., Kutufà, N.: Progress on the development of an iodine-fed hall effect thruster. IEPC-2017-418, 35th international electric propulsion conference, Atlanta, GA, USA (2017)

16. Martinez Martinez, J., Rafalskyi, D., Aanesland, A.: Iodine-a game-changing propellant for plasma based electric propulsion. SP2018-501, 6th Space propulsion conference, Seville, Spain (2018)

17. Official NIST web site: https://webbook.nist.gov/chemistry/ (2020). Accessed 9 Sept 2020

18. Tsay, M., Frongillo, J., Hohman, K.: Iodine-fueled mini RF ion thruster for CubeSat applications. IEPC-2015-273, 34th international electric propulsion conference, Kobe, Japan (2015)

19. Polzin, K.A., Peeples, S.R., Seixal, J.F., Mauro, S.L., Lewis, B.L., Jerman, G.A., Calvert, D.H., Dankanich, J., Kamhawi, H., Hickman, T.A., Szabo, J., Pote, B., Lee, L.: Propulsion system development for the iodine satellite (iSAT) demonstration mission. IEPC2015-09, 34th international electric propulsion conference, Kobe, Japan (2015)

20. Tsay M., Frongillo, J., Hohman, K., Malphrus, B.K.: LunaCube: a deep space $6 \mathrm{U}$ CubeSat with mission enabling ion propulsion technology. SSC15-XI-1, 29th AIAA/USU small satellites conference, Logan, UT, USA (2015)

21. Manente, M., Trezzolani, F., Magarotto, M., Fantino, E., Selmo, A., Bellomo, N., Toson, E., Pavarin, D.: REGULUS: a propulsion platform to boost small satellite missions. Acta Astronaut. 157, 241-249 (2019)

22. Official Technology for Propulsion and Innovation web site: http:// www.t4innovation.com/ (2020). Accessed 9 Sept 2020

23. Santoni, F., Piergentili, F., Graziani, F.: The UNISAT program: lessons learned and achieved results. Acta Astronaut. 65(1-2), 54-60 (2009)

24. Cannat, F., Laeur, T., Jarrige, J., Chabert, P., Elias, P.Q., Packan, D.: Optimization of a coaxial electron cyclotron resonance plasma thruster with an analytical model. Phys. Plasmas 22(5), 053503 (2015)

25. Magarotto, M., Pavarin, D.: Parametric study of a cathode-less radio frequency thruster. IEEE Trans. Plasma Sci. 48(8), 2723$2735(2020)$

26. Magarotto, M., Melazzi, D., Pavarin, D.: Study on the influence of the magnetic field geometry on the power deposition in a helicon plasma source. J. Plasma Phys. (2019). https://doi.org/10.1017/ S0022377819000473

27. Chen, F.F.: Helicon discharges and sources: a review. Plasma Sources Sci. Technol. 24(1), 014001 (2015)

28. Magarotto, M., Melazzi, D., Pavarin, D.: 3D-VIRTUS: Equilibrium condition solver of radio-frequency magnetized plasma discharges for space applications. Comput. Phys. Commun. 247, 106953 (2020)

29. Ahedo, E., Merino, M.: Two-dimensional supersonic plasma acceleration in a magnetic nozzle. Phys. Plasmas 17(7), 073501 (2010)

30. Magarotto, M., et al.: Numerical model of a helicon plasma thruster. IEEE Trans. Plasma Sci. 48(4), 835-844 (2020)

31. Gallina, G., et al.: Enhanced biDimensional pIc: an electrostatic/ magnetostatic particle-in-cell code for plasma based systems. J. Plasma Phys. (2019). https://doi.org/10.1017/S00223778190002 05

32. Pavarin, D., Lucca Fabris, A., Trezzolani, F., Manente, M., Faenza, M., Ferri, F., Selmo, A., Katsonis, K., Berenguer, C.: Low power RF plasma thruster experimental characterization. AIAA 2012-4191, Proceedings of the 48th AIAA/ASME/SAE/ASEE joint propulsion conference and exhibit, Atlanta, GA, USA (2012)

33. Manente, M. et al.: REGULUS: know-how acquired on iodine propellant. IEPC-2019-419, 36th international electric propulsion conference, Wien, AT (2019)

34. Trezzolani, F., Magarotto, M., Manente, M., Pavarin, D.: Development of a counterbalanced pendulum thrust stand for electric propulsion. Measurement 122, 494-501 (2018)

35. Takahashi, K.: Helicon-type radiofrequency plasma thrusters and magnetic plasma nozzles. Rev. Mod. Plasma Phys. 3(1), 1-61 (2019)

36. Manente, M. et al.: REGULUS: iodine fed plasma propulsion system for small satellites. IEPC-2019-417, 36th International electric propulsion conference, Wien, AT (2019)

37. Spacecraft mechanical loads analysis handbook, European Cooperation for Space Standardization, ECSS-E-HB-32-26A (2021)

38. Hevner, R., Holemans, W., Puig-Suari, J., Twiggs, R.: An advanced standard for cubesats. SSC11-II-3, proceedings of the 25th AIAA/USU small satellites conference, Logan, UT, USA (2011)

39. Cubesat standards website: www.cubesat.org/ (2020). Accessed: 9 Sept 2020

40. Official APWorks web site: https://apworks.de/en/scalmalloy/ (2020). Accessed 9 Sept 2020

41. Official Ansys web site: https://www.ansys.com/ (2020). Accessed 9 Sept 2020

42. Official Octave web site: https://www.gnu.org/software/octave/ (2020) Accessed 9 Sept 2020

43. Official SPENVIS web site: https://www.spenvis.oma.be/ (2020). Accessed 9 Sept 2020

44. Sinclair, D., Dyer, J.: Radiation effects and COTS parts in SmallSats. SSC13-IV-3, 28th AIAA/USU small satellites conference, Logan, UT, USA (2013)

45. Mansutti, G., et al.: Modeling and design of a plasma-based transmit-array with beam scanning capabilities. Results Phys. 16, 102923 (2020)

46. Daykin-Iliopoulos, A., et al.: Characterisation of a thermionic plasma source apparatus for high-density gaseous plasma antenna applications. Plasma Sources Sci. Technol. 29(11), 115002 (2020)

47. De Carlo, P., et al.: Feasibility study of a novel class of plasma antennas for SatCom navigation systems. Acta Astronaut. 178, 846-853 (2021)

48. Magarotto, M., et al.: Numerical suite for gaseous plasma antennas Simulation. IEEE Trans. Plasma Sci. 49(1), 285-297 (2021)

49. Leens, F.: An introduction to I2C and SPI protocols. IEEE Instrum. Meas. Mag. 12(1), 8-13 (2009)

50. Official JTAG web site: https://www.jtag.com/. (2020) Accessed 9 Sept 2020

51. Official FreeRTOS web site: https://www.freertos.org/ (2020). Accessed 9 Sept 2020 
52. Stesina, F. et al.: Assesment of the impact of a miniaturized electric propulsion system on the CuneSat technologies. SP2020+1314,7 th space propulsion conference, on-line (2021)

53. Official ASTM International web site: https://webstore.ansi.org (2020) Accessed 9 Sept 2020

54. Sarrailh, P., et al.: Spis 5: new modeling capabilities and methods for scientific missions. IEEE Trans. Plasma Sci. 43(9), 2789-2798 (2015)

55. Li, M., et al.: On electron boundary conditions in PIC plasma thruster plume simulations. Plasma Sources Sci. Technol. 28(3), 034004 (2019)

56. Di Fede, S. et al.: Numerical simulation of the plume of a magnetically enhanced plasma thruster. SP2020+1-111, 7th space propulsion conference, on-line (2020)

57. Grondein, P., et al.: Global model of an iodine gridded plasma thruster. Phys. Plasmas 23(3), 033514 (2016)

58. Official International Reference Ionosphere web site: http://irimo del.org/. Accessed 9 Sept 2020
59. Szabo, J.J.: Fully kinetic numerical modeling of a plasma thruster. $\mathrm{PhD}$ dissertation, Massachusetts Institute of Technology, Aeronautics and Astronautics Department (2001)

60. Martinez-Sanchez, M., Navarro-Cavalle, J., Ahedo, E.: Electron cooling and finite potential drop in a magnetized plasma expansion. Phys. Plasmas 22(5), 053501 (2015)

61. Cichocki, F., et al.: Hybrid 3D model for the interaction of plasma thruster plumes with nearby objects. Plasma Sources Sci. Technol. 26(12), 125008 (2017)

62. Martegani, P., Joukova, A.: Debris collision alert system. 1st NEO and debris detection conference, Darmstadt, DE (2019)

Publisher's Note Springer Nature remains neutral with regard to jurisdictional claims in published maps and institutional affiliations. 